\title{
Tumorigenicity of cells transformed by adenovirus type 12 by evasion of T-cell immunity
}

\author{
R. Bernards, P. I. Schrier, A. Houweling, J. L. Bos \& A. J. van der Eb
}

Department of Medical Biochemistry, Sylvius Laboratories, State University, Wassenaarseweg 72, 2333 AL Leiden, The Netherlands

\section{Zijlstra \& C. J. M. Melief}

Department of Tumor Immunology, Central Laboratory of the Netherlands Red Cross Blood Transfusion Service, PO Box 9190 , Amsterdam, The Netherlands

Evidence is presented that cells transformed by adenovirus type 12 are oncogenic because they escape from T-cell immunity. This effect is brought about by reducing the expression of class I transplantation antigens and is a function of the protein translated from the $13 S \mathrm{mRNA}$, transcribed from early region 1a. These findings establish a novel mechanism by which transformed cells can acquire an oncogenic phenotype.

ADENOVIRUS-transformed rodent cells exhibit variable degrees of oncogenicity, depending on the adenovirus species used for transformation: rat cells transformed by the highly oncogenic adenovirus 12 (Ad12) are highly oncogenic in the syngeneic host ${ }^{1}$, whereas rat cells transformed by the nononcogenic adenoviruses, for example $\mathrm{Ad} 2$ and $\mathrm{Ad} 5$, are rarely tumorigenic in immunocompetent syngeneic rats ${ }^{2}$. It has been proposed that cells transformed by the non-oncogenic adenoviruses fail to form tumours because they are more antigenic than are cells transformed by oncogenic adenoviruses and therefore are more readily eliminated by the immune system of the host ${ }^{3}$. The main evidence supporting this hypothesis is that rat cells transformed by the non-oncogenic Ad2, which are non-oncogenic in immunocompetent rats, do form tumours in immunosuppressed rats ${ }^{4}$ and in congenitally athymic nude mice ${ }^{5}$.

The adenovirus genes responsible for transformation in vitro are localized in a region near the left end of the viral genome, in a 4-kilobase $(\mathrm{kb})$ DNA segment which comprises the first region of the genome to be expressed in lytic infection, and therefore designated early region $1(E 1)$. This region consists of two transcriptional units, E1a and $E 1 b$ (ref. 6). We have recently demonstrated that the difference in oncogenic potential between Ad5- and Ad12-transformed cells in nude mice is determined by the large tumour antigen specified by subregion $E 1 b^{7,8}$. We show here that the identity of the E1a subregion determines the susceptibility of transformed cells to the cellular immune system, that is, rat cells expressing Ad5 E1a are highly susceptible to cytotoxic $\mathrm{T}$ cells and so are only oncogenic in immunodeficient animals, whereas cells expressing Ad12 E1a have a reduced susceptibility for T-killer cells and hence are oncogenic. By using a set of cell lines transformed by mutant $E 1$ regions we show that the inactivation of major histocompati- bility complex (MHC) class I gene expression is a function of the product of the Ad12 13S E1a mRNA.

\section{Oncogenicity of Ad-transformed cells}

We have recently reported the construction of two Ad5-Ad12 hybrid early region 1 plasmids: one, pAd512, consisting of the $E 1 a$ region of non-oncogenic $\mathrm{Ad} 5$ and the $E 1 b$ region of highly oncogenic Ad12; and the other, pAd125, consisting of Ad12 $E 1 a$ and $\operatorname{Ad} 5 E 1 b$. These plasmids were used to transform primary baby rat kidney (BRK) cells and the oncogenicity of the resulting transformed cell lines was tested in nude mice ${ }^{7}$. Comparison of the oncogenic potential of the various transformed cell lines in immunocompetent rats and in immunodeficient nude mice (Table 1 ) revealed a major contribution of region E1a in oncogenicity: cells expressing Ad5 E1a (Ad5 E1- and pAd512-transformed cells) were both oncogenic in nude mice, but completely failed to induce tumours in immunocompetent rats. On the other hand, cells expressing Ad12 E1a (Ad12 E1- and pAd125-transformed cells) manifested a similar oncogenic potential both in immunodeficient nude mice and in immunocompetent rats. The different results obtained in the mouse and rat assay systems for oncogenicity are most readily explained in terms of a thymusmediated immune response, which is present in the immunocompetent rat but absent in the nude mouse. The finding that pAd512-transformed cells were also highly oncogenic in immunodeficient nude rats (Table 1) is consistent with this explanation. Therefore, these data suggest that cells expressing Ad5 E1 $a$ are susceptible to elimination by T cells and so are non-oncogenic in the immunocompetent rat, in spite of their limited (Ad5 E1) or high (Ad512) oncogenicity in nude mice. On the other hand, cells expressing Ad12 E1a seem to escape

Table 1 Oncogenicity of adenovirus-transformed cells in syngeneic rats

Plasmids used for
transformation
pAd5XhoC
pAd12RIC
pAd512
pAd125
p51212

$\begin{array}{cc}\text { Expression in transformed cell } \\ \text { E1a } & \text { E1b } \\ 5 & 5 \\ 12 & 12 \\ 5 & 12 \\ 12 & 5 \\ 5+12 & 12\end{array}$

$10^{7}$ transformed cells were injected subcutaneously into 4-day-old Wag-Rij rats or in adult athymic nude Wag-Rij rats. The cell lines used and the oncogenicity of these lines in athymic nude mice have been described before ${ }^{7}$, with the exception of p51212-transformed cells, which express the Ad5 and Ad12 E1a regions simultaneously at comparable levels plus the Ad12 E1b region (Fig. 3). For each type of transformed cells at least three independently isolated cells lines were used. Numbers in parentheses indicate the total number of animals with tumours/total number of animals injected. Average latent periods were: ${ }^{*}, 6$ weeks; $†, 4$ months; $\ddagger, 3$ months. ND, not done.

\begin{tabular}{ccc}
\multicolumn{3}{c}{$\%$ Oncogenicity of transformed cell in } \\
Nude mice & Syngeneic rats & Nude rats \\
$50(15 / 31)$ & $0(0 / 51)$ & ND \\
$100(23 / 23)$ & $100(18 / 18)^{*}$ & ND \\
$100(18 / 18)$ & $0(0 / 26)$ & $100(6 / 6) \ddagger$ \\
$10(2 / 19)$ & $10(6 / 60) \dagger$ & ND \\
$100(12 / 12)$ & $0(0 / 18)$ & ND
\end{tabular}




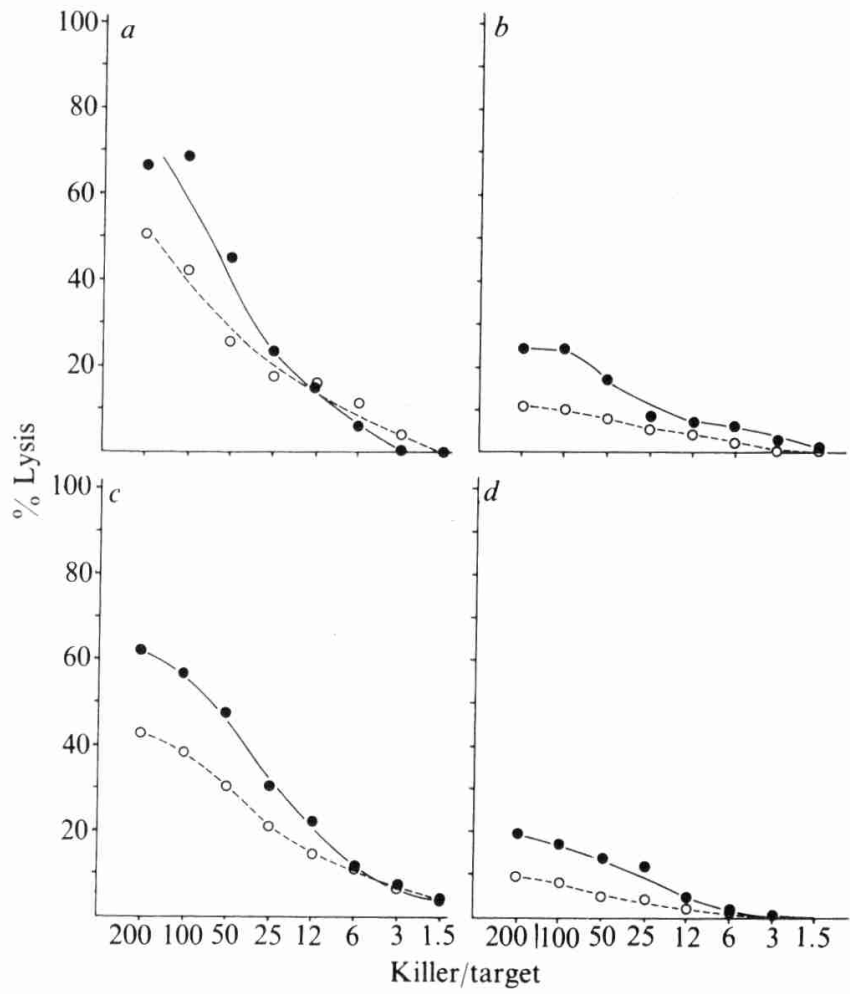

Fig. 1 Susceptibility of adenovirus-transformed Wag-Rij cells to allogeneic CTLs. For every type of transformed target cell, the lytic activity of CTLs is shown against two independently derived cell lines. $a$, Cells transformed by Ad5 region E1, pAd5XhoC (ref. 7). $b$, Cells transformed by Ad12 region $E 1$, pAd12RIC (ref. 26). $c$, Cells transformed by a plasmid containing Ad5 E1a and Ad12 E1b, pAd512 (ref. 7). d, Cells transformed by a plasmid containing Ad12 E1a and Ad5 E1b, pAd125 (ref. 7). Target cells were labelled with $100 \mu \mathrm{Ci}$ of ${ }^{51} \mathrm{Cr}$-labelled sodium chromate for $30 \mathrm{~min}$ at $37^{\circ} \mathrm{C}$, and washed three times to remove free chromium. To raise anti- $R T 1^{u}$ allogeneic CTLs, spleen cells of ACI rats ( $R T 1^{a}$ haplotype) were cultured for 6 days with irradiated $(2,500 \mathrm{rad})$ lymphocytes derived from the spleens of Wag-Rij rats at a ratio of three responders to one stimulator as described previously ${ }^{27}$. Reactivity of CTL was assayed by measuring ${ }^{51} \mathrm{Cr}$ release of triplicate samples of targets in microtitre plates for $4 \mathrm{~h}$ at $37^{\circ} \mathrm{C}$, using $10^{4}$ targets per well. Per cent lysis was calculated according to $(E-C) /(M-C) \times 100$, where $E=$ counts released from targets in the presence of CTLs, $C=$ counts released from targets in the absence of CTLs, $M=$ counts released from targets lysed with $5 \%$ Triton.

T-cell-mediated destruction since they are equally oncogenic in the nude mice and in the syngeneic rats.

\section{Susceptibility to cytotoxic $\mathbf{T}$ cells}

In the accompanying paper ${ }^{9}$ we show that rat cells expressing the Ad12 E1a region have a reduced expression of the heavy chain of the class I transplantation antigens. As foreign (viral) antigens are only recognized by cytoxic T lymphocytes (CTLs) in the context of MHC class I molecules ${ }^{10}$, reduced expression of class I polypeptides could explain the escape of cells expressing Ad12 E1a from the T-cell-mediated immune response. To investigate the extent to which reduced expression of class I molecules in cells expressing Ad12 E1a reduces susceptibility to CTLs, we have measured lysis of Ad5- and Ad12-transformed cells and of the Ad5-Ad12 recombinant cell lines by allogeneic CTLs generated in primary mixed lymphocyte culture. Such CTLs have been shown to be mainly directed against class I molecules ${ }^{1-13}$. All adenovirus-transformed cells used in the oncogenicity studies were derived from kidneys of the highly inbred strain Wag- $\mathrm{Rij}$ ( $R T 1^{u}$ haplotype). To raise anti- $R T 1^{u}$ allogeneic CTLs, spleen cells of ACI rats $\left(R T 1^{a}\right.$ haplotype) were cultured in vitro with irradiated spleen cells from Wag-Rij

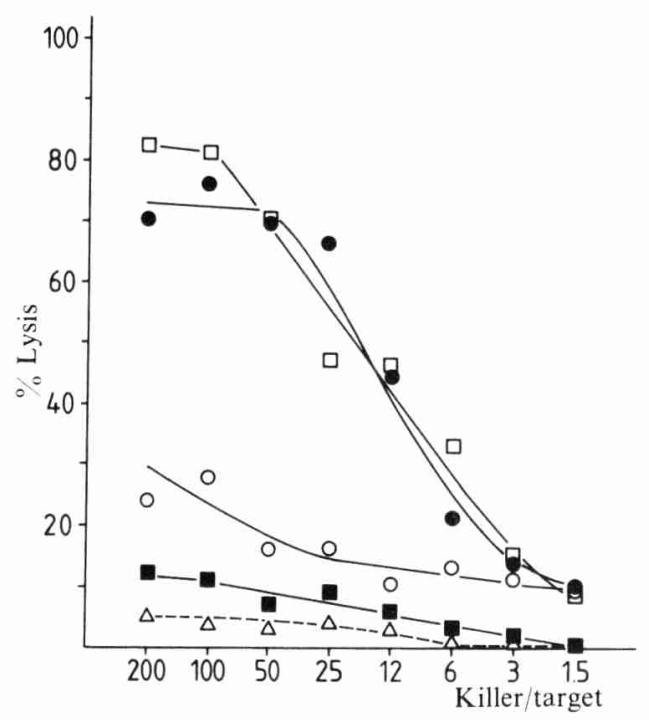

Fig. 2 Reactivity of anti- $R T 1^{n}$ allogeneic CTL against Ad5- and Ad12-transformed Brown Norway rat cells. $\square$, Ad5-transformed cell lines; $O$, $\mathbf{E}$, Ad12-transformed cell lines; $\triangle$, Ad12transformed Wag-Rij line. Anti-RT $1^{n}$ killer cells were generated in 6-day mixed lymphocyte cultures. Responder strain was the Wag-Rij rat. Stimulators were irradiated Brown Norway Spleen cells. For further explanatory notes, see Fig. 1 legend.

rats. The reactivity of these allogeneic CTLs against a set of adenovirus-transformed cells shows a strong response by the allogeneic CTLs against both Ad5 E1-transformed cells (Fig. 1a) and pAd512-transformed cells (Fig. 1c). The same CTLs have a low, but reproducible, reactivity against both Ad12 E1(Fig. $1 b$ ) and pAd125- (Fig. 1d) transformed cells. These data are in excellent agreement with our studies on oncogenicity of these cells in immunodeficient and immunocompetent animals, from which we conclude that cells expressing Ad12 E1a escape T-cell-mediated immune response (Table 1).

A similar difference in susceptibility to allogeneic CTLs was observed for Ad5- and Ad12-transformed Brown Norway rat cells ( $R T 1^{n}$ haplotype) as for Ad5- and Ad12-transformed Wag-Rij cell lines (Fig. 2). It seems likely, therefore, that suppression of class I antigen expression by Ad12 region E1a is, at least in the rat system, a rather general phenomenon. A low, but reproducible, CTL response was found against the Ad12-transformed rat cells derived from the two different inbred rat strains tested. Indeed, long exposure of autoradiographs of immunoprecipitations with anti-class I antisera revealed small amounts of class I antigens in these cells. The low residual activity of the mixed lymphocyte culture-activated cells is therefore probably due to specific CTL activity. The specificity of CTLs was shown by the fact that responder-type target cells were not lysed (Fig. 2) and only low specific lysis of unrelated target cells was detected (not shown).

\section{Suppression of class I antigen production}

The Ad12 E1a region encodes two co-terminal mRNAs of $12 \mathrm{~S}$ and $13 \mathrm{~S}$ respectively, differing only in the amount of RNA removed internally by splicing ${ }^{14}$. To investigate which of these two mRNAs, if not both, encodes the inactivating function, we have investigated the expression of class I transplantation antigens in two cell lines transformed by Ad12 region $E 1$ plasmids carrying specific mutations in the E1a region. The results (Fig. 3 ) show that expression of class I antigens is not reduced in cells transformed by pSVR7, a mutant plasmid with a single nucleotide deletion affecting the $13 \mathrm{~S}$ mRNA only ${ }^{15}$ (lane 1), or in cells transformed by pSVR11, a mutant plasmid with a 106-base pair (bp) deletion affecting both E1a mRNA species (lane 2). Thus the Ad12 gene product responsible for the reduced expression of class I antigens is encoded by the $13 \mathrm{~S}$ E1a mRNA. 


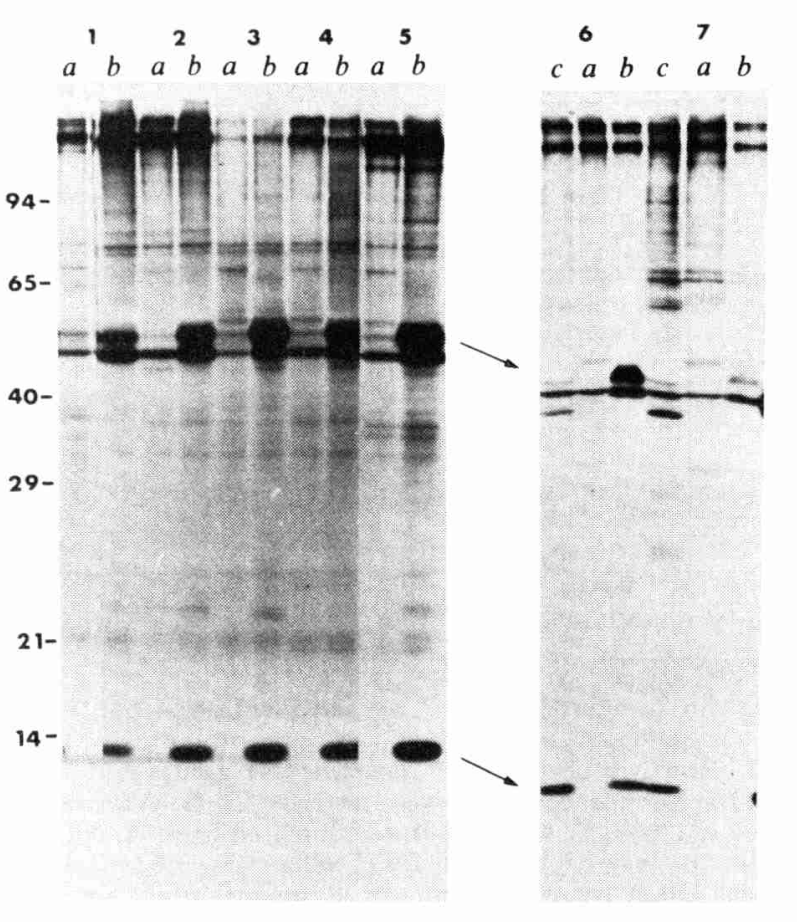

Fig. 3 Immunoprecipitation of ${ }^{35} \mathrm{~S}$-methionine-labelled cell extracts of adenovirus-transformed cells with anti-MHC class I antisera. The sera used were either normal rat serum (lanes $a$ ) or Lewis anti-Wag-Rij alloantiserum (lanes $b$ ), or an anti-human $\beta_{2}$-microglobulin antiserum (lanes $c$ ). The cell lines used were transformed by: pSVR7 (ref. 15), lane 1; pSVR11 (ref. 15), lane 2; pLT12 (ref. 8), lane 3; pST12 (ref. 8), lane 4; p51212, lane 5; p512pm975, lane 6; p512HL1007, lane 7. Positions of the molecular weight markers are indicated. Procedures for immunoprecipitation were as previously described ${ }^{7}$. p51212 was constructed by inserting the Ad12 EcoRI C fragment in pAd5 E1a (ref. 7). p512pm975 was made by cloning the Ad12 EcoRI $\mathrm{C}$ fragment into pEKpm975 (ref. 28). p512HL1007 was constructed by inserting a 10-bp HindIII linker at the single SmaI site of pAd5 E1a (position 1,007 of the Ad5 sequence) and subsequent insertion of the Ad12 EcoRI C fragment in the mutagenized pAd5 E1a plasmid. The identity of the mutation was confirmed by DNA sequencing. p512pm975-transformed cells express the Ad12 region $E 1$ genes plus the Ad5 13S E1a mRNA, p512HL1007transformed cells express Ad12 region E1 genes, the Ad5 $12 \mathrm{~S}$ E1a mRNA gene product plus a truncated 13S E1a protein. Correct expression of the mutagenized E1a regions in transformed cells was confirmed in $S_{1}$ nuclease experiments (not shown).

To investigate the mechanism by which Ad12 region E1a switches off the MHC class I genes in transformed cells, we have studied the activity of the genes in cell lines which simultaneously express the E1a regions of both Ad5 and Ad12 (ref. 8). The results show that three independently isolated cell lines, coexpressing Ad5 and Ad12 region E1a, have normal levels of class I antigens (Fig. 3, lanes 3-5), indicating that expression of Ad5 E1a can block the inactivating functions of Ad12 E1a. Co-expression of the Ad5 13S E1a mRNA and region E1a of Ad12 allowed production of class I antigens (see Fig. 3 legend), while cells co-expressing the Ad5 12S. E1 $a$ mRNA and E1a of Ad12 did not have detectable levels of class I antigens (Fig 3, lanes 6,7$)$. Thus, the Ad5 $13 \mathrm{~S}$ E1a mRNA is responsible for the dominant effect of Ad5 over Ad12.

Taking advantage of this finding we tested directly the relationship between the reduced class I expression and the highly oncogenic phenotype of the Ad12-transformed cells by using the recombinant plasmid p51212 (see Fig. 3 legend). Rat cells transformed by p51212 express all Ad12 transforming genes plus region E1a of Ad5 (not shown). They produce normal amounts of class I antigens (Fig. 3, lane 5), are sensitive to allogeneic CTLs (not shown) and are highly oncogenic in nude mice, but fail to induce tumours in syngeneic rats (Table 1). Thus, cells expressing all Ad12 transforming genes are nontumorigenic in syngeneic rats if the function which inactivates MHC class I expression is blocked. This strongly suggests that the unique capacity of Ad12 E1a virtually to abolish expression of MHC class I genes must be the molecular basis for the difference in oncogenicity between Ad5- and Ad12-transformed cells.

\section{Discussion}

Immunological defence mechanisms, mediated by $\mathrm{T}$ cells, are considered to play a crucial part in the elimination of virusinduced tumours ${ }^{13,16,17}$. For example, T-cell-deficient nude mice show a markedly increased susceptibility to various virusinduced tumours compared with normal mice ${ }^{5,18,19}$. It has been shown that CTLs only recognize a viral antigen in the context of self-MHC class I antigens ${ }^{10}$. Thus, CTLs have combined specificity for viral antigens and class I MHC molecules, while. T-helper cells have combined specificity for antigens and class II MHC molecules. The adenovirus-mediated modulation of MHC class I expression, as described in the accompanying paper ${ }^{9}$, offers an ideal system to study the functional role of CTLs in tumour rejection. As a first step we have shown that the decreased expression of class I antigens in Ad12-transformed cells results in a dramatic decrease in susceptibility to allogeneic CTLs, which are known to be directed against class I molecules. Of course, this finding is no direct proof for decreased susceptibility to virus-specific CTLs in vivo. However, it seems likely that at least the same amount of class I molecules is required for lysis by syngeneic class I-restricted CTLs as for lysis by allogeneic CTLs, for allogeneic CTLs directly recognize class I molecules, while virus-specific CTLs probably recognize conformational determinants on class I molecules, created by interaction with viral antigens at the cell surface. Indeed, we have found that Ad5-transformed BRK cells induced an excellent adenovirus-specific secondary CTL response in vitro, while the same cells transformed by Ad12 practically failed to do so (R.B., unpublished).

Our results indicate that CTLs also have a lytic activity against Ad12-transformed cells. The oncogenic phenotype of Ad12transformed cells might therefore not be due to a complete absence of CTL reactivity against such cells, but rather to a sufficiently reduced susceptibility to CTLs to allow the cells to evade elimination. Thus, on the basis of the combined in vivo and in vitro findings we propose that adenovirus-transformed cells expressing the Ad5 E1a region are eliminated in the immunocompetent host by an efficient CTL response. Hence, these cells are only oncogenic in immunodeficient animals such as nude mice ${ }^{5,7}$, nude rats (Table 1 ) and immunosuppressed rats $^{4}$. On the other hand, cells expressing Ad12 E1a are equally oncogenic in immunocompetent and in T-cell deficient animals (Table 1), apparently because these cells lack sufficient MHC class I antigens in the plasma membrane to be eliminated by specific CTLs (Figs 1, 2).

At this stage we cannot exclude that class II-restricted T cells also have a reduced response against Ad12-transformed cells compared with Ad5-transformed cells. However, both Ad5and Ad12-transformed cells induce high levels of IgG antibodies in immunized animals. Since production of antibodies requires class II-restricted T-helper cells, Ad12 probably does not interfere with class II-restricted immune response.

We have presented evidence that the gene product of the $13 \mathrm{~S}$ E1a mRNA of Ad12 is responsible for the absence of the heavy chain of class I antigens. We have recently shown that the same Ad12 E1a gene product is required to activate the Ad12 E1b promoter ${ }^{20}$. A similar activating function has also been demonstrated for the Ad5 E1a region, both for activation of region $E 1 b$ and other early regions of the viral genome $\mathrm{e}^{21-24}$, as well as for a cell encoded heat-shock protein ${ }^{25}$. It has been proposed that the E1a gene product manifests its activating function through the inactivation of a cellular repressor-like protein ${ }^{23}$. 
Therefore, the simplest possible model explaining both the activating and the inactivating functions of the polypeptide encoded by the $13 \mathrm{~S}$ Ad12 E1a mRNA is that it activates a cellular gene (via inactivation of a repressor) which itself has a repressing effect on other cellular genes. However, the Ad12 SVR7 mutant plasmid, which carries a single nucleotide deletion in the region encoding the Ad12 13S E1a mRNA, does not switch off the activity of class I genes in transformed cells, but has retained the capacity to activate the Ad12 E1b promoter ${ }^{15}$. That we can separate the activating and inactivating functions of the Ad12 E1a gene product in the SVR7 mutant suggests that these two functions are not manifested through an interaction with the same cellular control factor.

Finally we have shown that rat cells expressing the Ad5 and Ad12 E1a regions simultaneously do express normal levels of

Received 16 June; accepted 2 September 1983

Freeman, A. E., Black, P. H. Wolford, R. \& Huebner, R. J. J Virol 1, 362-367 (1967) Freeman, A. E. et al. Proc. natn. Acad. Sci. U.S. A. 58, 1205-1212 (1967).

McAllister, R. M., Nicholson, M. O., Lewis, A. M., MacPherson, I. \& Huebner, R. J. J. gen. Virol. 4, 29-36 (1969)

Gallimore, P. H. J. gen. Virol. 16, 99-102 (1972).

Gallimore, P. H., McDougall, J. K. \& Chen, L. B. Cell 10, 669-678 (1977).

Flint, S. J. in Molecular Biology of Tumor Viruses II: DNA Tumor Viruses 2nd edn (ed.

Tooze, J.) 547-576 (Cold Spring Harbor Laboratory, New York, 1980).

Bernards, R., Houweling, A., Schrier, P. I., Bos, J. L. \& Van der Eb, A. J. Virology 120, $422-432$ (1982)

Bernards, R., Schrier, P. I., Bos, J. L. \& Van der Eb, A. J. Virology 127, 45-54 (1983).

Schrier, P. I., Bernards, R., Vaessen, R. T. M. J., Houweling, A. \& Van der Eb, A. J. Nature 305, 771-775 (1983)

10. Zinkernagel. R. M. \& Dohenty, P. C. Adv. Immun. 27, 51-177 (1979).

11. Bevan, M. J. Proc natn. Acad Sci. U.S. A. 74, 2094-2098 (1977).

12. Nabholz, M. et al. Eur. J. Immun. 4, 378-387 (1974)

3. Melief, C. J. M. \& Schwartz, R. S. in Cancer: a Comprehensive Treatise Vol. 1 (ed. Becker,

F. F.) 121-159 (Plenum, New York, 1975). class I antigens. Thus, Ad5 E1a can prevent Ad12 E1a from inactivating the class I genes. Such a dominant effect suggests that Ad5 and Ad12 E1a products are competing for binding to the same regulatory factor. The finding that the Ad5 protein responsible for the dominant effect is structurally closely related to the Ad12 product is consistent with such a competitive binding model. Clearly, the next step should be to unravel the mechanism by which the Ad12 E1 $a$ product interacts with the class I genes. Studies in this direction with cloned murine class I genes are in progress.

This work was supported in part by the Netherlands Organization for the Advancement of Pure Research (ZWO) through the Foundation for Fundamental Medical Research (FUNGO). We thank Dr A. J. Berk for the gift of pEKpm975 and Drs L. C. Paul and L. Vaessen for rat alloantisera.

14. Perricaudet, M. le Moullec, J., Tiollais, P. \& Petterson, U. Nature 288, 174-176 (1980). 15. Bos, J. L. et al. Virology (in the press).

16. Stutman, C. Adv. Cancer Res. 22, 261-422 (1975).

17. Klein, G. Cancer 45, 2486-2499 (1980).

18. Shin, S., Freedman, V. H., Risser, R. \& Pollack, R. Proc. natn. Acad. Sci. U.S.A. 72, 4435-4439 (1975).

19. Shin, S. Meth. Enzym. 58, 370-379 (1979).

20. Bos, J. L. \& ten Wolde-Kraamwinkel, H. C. EMBO J. 2, 73-76 (1983).

21. Jones, N. \& Shenk, T. Proc, natn. Acad. Sci. U.S.A. 76, 3665-3669 (1980).

22. Berk, A. J., Lee, F., Harrison, T., Williams, J. \& Sharp, P. H. Cell 17, 935-944 (1979).

23. Nevins, J. R. Cell 26, 213-220 (1981).

24. Ricciardi, R. P., Jones, R. L., Cepko, C. L., Sharp, P. A. \& Roberts, B. E. Proc. natn Acad. Sci. U.S.A. 78, 6121-6125 (1981).

25. Nevins, J. R. Cell 29, 913-919 (1982).

26. Bos, J. L. et al. Cell 27, 121-131 (1981)

27. Melief, C. J. M., de Waal, L. P., Van der Meulen, M. Y., Melvold, R. W. \& Kohn, H. I. J exp. Med 151, 993-1013 (1980)

28. Montell, C., Fisher, E. F., Caruthers, M. H. \& Berk, A. J. Nature 295, 380-384 (1982).

\section{Expression of recessive alleles by chromosomal mechanisms in retinoblastoma}

\section{W. K. Cavenee* ${ }^{*}$, T. P. Dryja ${ }^{\dagger}$, R. A. Phillips ${ }^{\ddagger}$, W. F. Benedict ${ }^{\S}$, R. Godbout ${ }^{\ddagger}$, B. L. Gallie ${ }^{\ddagger}$, A. L. Murphree", L. C. Strong ${ }^{\S}$ \& R. L. White ${ }^{*}$}

* Howard Hughes Medical Institute and Department of Cellular Viral and Molecular Biology, University of Utah School of Medicine, Salt Lake City, Utah 84132, USA

$\dagger$ Massachusetts Eye and Ear Infirmary, Harvard Medical School, Boston, Massachusetts 02114, USA

$\ddagger$ The Ontario Cancer Institute and University of Toronto, Toronto, Canada M4X $1 \mathrm{~K} 9$

$\S$ The Clayton Ocular Oncology Center, and the Division of Hematology/Oncology, Childrens Hospital of Los Angleles, and the Department of Pediatrics, University of Southern California School of Medicine, Los Angeles, California 90027, USA

$\|$ The Clayton Ocular Oncology Center, and the Division of Opthalmology, Childrens Hospital of Los Angleles, and the Department of Pediatrics, University of Southern California School of Medicine, Los Angeles, California 90027, USA

$\S$ Department of Pediatrics, M. D. Anderson Hospital and Tumor Institute, Houston, Texas 77030, USA

Inheritance of a mutation at the Rb-1 locus, which has been mapped to band q14 of human chromosome 13, results in predisposition to retinoblastoma. Cloned DNA segments homologous to arbitrary loci of human chromosome 13 and which reveal polymorphic restriction endonuclease recognition sequences, have been used to look for somatic genetic events that might occur during tumorigenesis. A comparison of constitutional and tumour genotypes from several cases indicates that tumorigenesis may result from the development of homozygosity for the mutant allele at the Rb-1 locus. The homozygosity in these cases results from mitotic nondisjunction, resulting in loss of the homologous wild-type chromosome, or from a mitotic recombination event.

RETINOBLASTOMA is one of several heritable childhood cancers. The disease exhibits both hereditary and sporadic occurrence, with the genetic form inherited as a highly penetrant autosomal dominant trait. Additionally, deletions of human chromosome 13 band q14 are occasionally associated with retinoblastoma ${ }^{1-3}$. Deletion ${ }^{4}$ and family studies ${ }^{5}$ have shown the genetic locus for the enzyme esterase $\mathrm{D}(E S-D)$, to be tightly

* Present address: Department of Microbiology and Molecular Genetics, University of Cincinnati Medical School, Cincinnati, Ohio 45267, USA. linked to $R b-1$, the retinoblastoma locus. Additional evidence supporting this map location comes from a family ${ }^{6}$ showing a balanced chromosomal translocation in unaffected carrier parents and some unaffected sibs and an unbalanced chromosome 13 deletion in affected individuals. The region of chromosome 13 which was deleted in the predisposed children was $\mathrm{q} 13.1-\mathrm{q} 14.5$, thus including the proposed $R b-1$ locus. This observation indicates that the deletion mutation of the $R b-1$ locus was recessive to two copies of the normal gene. It is possible, then, that the inherited retinoblastoma mutation is actually a recessive allele requiring subsequent inactivation of 